\title{
Novel superconducting rf structure for ampere-class beam current for multi-GeV energy recovery linacs
}

\author{
Z. Liu ${ }^{1,2}$ and A. Nassiri ${ }^{2}$ \\ ${ }^{1}$ Institute of Heavy Ion Physics, Peking University, Beijing, China \\ ${ }^{2}$ Advanced Photon Source, Argonne National Laboratory, 9700 S. Cass Avenue, Argonne, Illinois 60439, USA
}

(Received 12 August 2009; published 6 January 2010)

\begin{abstract}
Future ampere-class energy recovery linacs (ERLs) based on superconducting technology demand efficient damping of the higher-order modes in the superconducting radio-frequency (SRF) accelerating structures to achieve stable beam operation in multipass, multibeam ERLs. We propose a new and novel SRF structure that is extremely efficient in damping the higher-order modes of SRF structures for ERLs. Initial simulation results indicate extremely good and efficient damping of the dipole and the quadrupole modes that determine the beam breakup threshold of the superconducting structures. The proposed new structure has the added benefit of having simpler fabrication steps with potential fabrication cost savings.
\end{abstract}

DOI: 10.1103/PhysRevSTAB.13.012001

PACS numbers: 29.20.Ej

\section{INTRODUCTION}

Multipass, multibunch beam breakup (BBU) can limit beam current in energy recovery linacs (ERLs). Multipass, multibunch instabilities driven by high-impedance dipole and quadrupole modes will result in beam breakup. Therefore, to increase the BBU threshold, one needs to damp the higher-order modes (HOMs) efficiently to avoid beam instabilities. Traditionally, large iris and beam pipe diameters [1-5] are employed for the removal of HOM power using ferrite absorbers in the cavity beam pipe. Via a combination of enlarging the cavity beam pipe, optimizing cavity cell geometry, and designing efficient HOM absorbers, the BBU threshold can achieve hundreds of milliamperes $[1-4,6]$. However, in order to achieve proper HOM damping, one must choose a smaller number of cells to avoid trapped modes inside the structure, which have an unwanted impact on achieving a high accelerator packing factor. Using a smaller number of cells will result in an overall increased accelerator length and cost of the linac.

For multi-MeV ERLs, the design goal is to achieve ampere-class beam current. For example, the Brookhaven National Laboratory (BNL) R\&D ERL $[7,8]$ is composed of four five-cell cavities working at $703.75 \mathrm{MHz}$ with a final energy of $54 \mathrm{MeV}$. The calculated BBU threshold is larger than $1 \mathrm{~A}$. The JLab ERL [6] is composed of six cavities operating at $1.497 \mathrm{GHz}$ with a maximum final beam energy of $60 \mathrm{MeV}\left(E_{\text {acc }}=16.7 \mathrm{MV} / \mathrm{m}\right)$ utilizing a $10-\mathrm{MeV}$ injector. The averaged $\mathrm{BBU}$ threshold for this machine is $0.95 \mathrm{~A}$.

For multi-GeV ERLs, the designed BBU threshold is in the $100-\mathrm{mA}$ range with cavities operating between 1.3 and 1.5 GHz. For example, the High Energy Accelerator Research Organization (KEK) 5-GeV ERL, which is designed to operate at $1.3 \mathrm{GHz}$ with nine-cell cavities, has a BBU threshold of $600 \mathrm{~mA} \mathrm{[1],} \mathrm{while} \mathrm{the} \mathrm{Cornell}$ University $1.3-\mathrm{GHz}, 5-\mathrm{GeV}$ single-pass ERL with seven- cell cavities based on the TESLA-type [9] cavity, has a BBU threshold of $100 \mathrm{~mA}$ [4]. For the proposed Argonne 7-GeV single-pass ERL based on five-cell superconducting cavities operating at $1.407 \mathrm{GHz}$, the predicted BBU threshold using code TDBBU [10] is $200 \mathrm{~mA}$.

There are no convincing results that indicate the existing multicell SRF cavities could truly achieve the ampere-class BBU threshold in multi-GeV ERLs. We have proposed and designed a new superconducting rf (SRF) cavity that meets the HOM damping requirements of multi-GeV, ampereclass ERLs. Our proposed design will mitigate issues associated with electron-beam welding at the high-field regions of the SRF cavities. It is known that welds at the high-field regions of the SRF cavities have resulted in unpredictable faults when operated with high fields at $2 \mathrm{~K}$ [11]. The proposed new structure avoids welds on high-field regions, thus significantly improving HOM damping in multicell cavities.

\section{CAVITY DESIGN}

An approximate formula of the threshold current in the presence of a single higher-order mode, with the assumption that the transverse HOMs behave independently and do not interfere with each other, is [12-15]

$$
I_{\mathrm{th}}=-\frac{2 c^{2}}{e\left(\frac{R}{Q}\right)_{\lambda} Q_{\lambda} \omega_{\lambda}} \frac{1}{T_{12}^{*} \sin \omega_{\lambda} t_{r}}
$$

and

$$
T_{12}^{*}=T_{12} \cos ^{2} \theta_{\lambda}+\frac{T_{14}+T_{32}}{2} \sin 2 \theta_{\lambda}+T_{34} \sin ^{2} \theta_{\lambda} .
$$

Here $c$ is the speed of light, $e$ is the elementary charge, $\lambda$ is the mode number, $(R / Q)_{\lambda}$ is the shunt impedance (in units 
of $\Omega$ ), $Q_{\lambda}$ is the quality factor, $\omega_{\lambda}$ is the HOM frequency, $\theta_{\lambda}$ is the polarization angle from the $x$ direction, $t_{r}$ is the bunch return time, and the matrix $T$ describes how a transverse momentum is transported to a transverse displacement after one turn.

From Eq. (1) we know that the BBU threshold is determined by the cavity intrinsic parameter $(R / Q)_{\lambda} \cdot Q_{\lambda}$ and the linac optic transfer matrix. A goal of the cavity design is to minimize $(R / Q)_{\lambda} \cdot Q_{\lambda}$ value in order to achieve a higher BBU threshold. The quantity $(R / Q)_{\lambda} \cdot Q_{\lambda}$ above is the sum of the impedances of $\lambda$ mode contributed by each cavity; therefore, the number of cavities will greatly influence the BBU threshold limit, since the BBU threshold is inversely proportional to the number of cavities. Assuming that all the cavities have the same accelerating gradient and the same $(R / Q)_{\lambda} \cdot Q_{\lambda}$, higher-energy ERLs will require a larger number of cavities, thus limiting the operation of the linac to a low-current BBU threshold. The only way to increase the BBU threshold to a higher beam current for a multi-GeV, ampere-class ERL is to decrease the $(R / Q)_{\lambda}$. $Q_{\lambda}$ of the transverse HOM to an acceptable level. Our proposed design accomplishes this by incorporating three low-cutoff, equally spaced waveguide branches $\left(120^{\circ}\right.$ apart) into the body of the cavity along the beam axis (see Fig. 1). This structure has a lower $(R / Q)_{\lambda} \cdot Q_{\lambda}$ of the dipole and the quadrupole modes-more than 2 and 7 orders of magnitude, respectively, than the Cornell sevencell cavity [4].

The new cavity structure is based on a previously designed high-current, five-cell superconducting cavity that is being considered as part of a feasibility study for the Advanced Photon Source (APS) ERL [16,17]. Although the cavity shape of the new structure has not yet been optimized, it already exhibits excellent damping of the dipole and quadrupole modes.

This cavity is made up of three equal parts; each part can be deep drawn out of a single niobium sheet. Figure 1 shows a three-dimensional view of the cavity. The Argonne five-cell, high-current cavity [2] shape was adopted for the center portion of the new cavity. The center cavity is slotted in the longitudinal direction (along the beam axis) at three places, with an equal azimuthal separation of $120^{\circ}$.

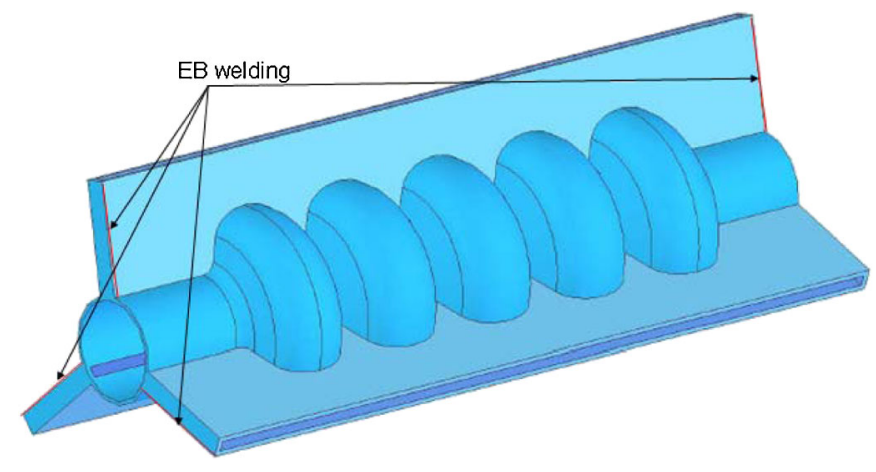

FIG. 1. (Color) Slotted cavity structure.

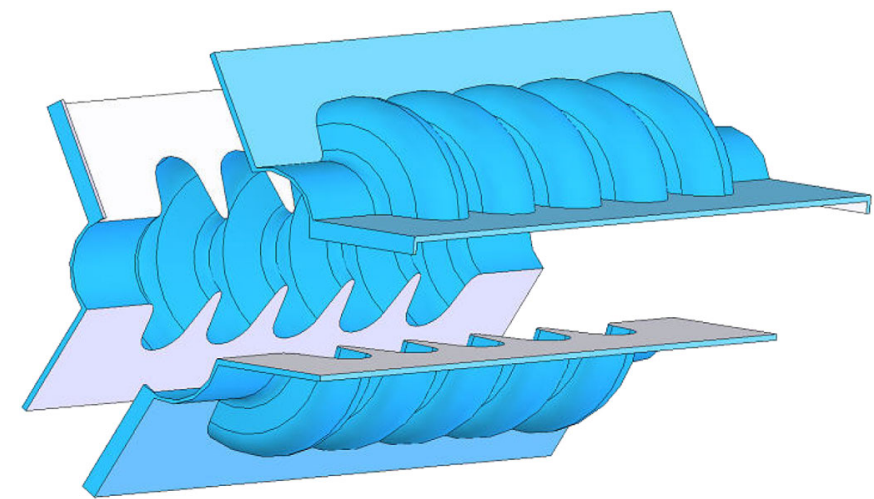

FIG. 2. (Color) Three equal parts of the slotted cavity.

Each slot is extended to form a wide-open waveguide, as shown in Fig. 1. Figure 2 shows the three equal parts of the cavity. The three parts of the cavity are electron-beam (EB) welded together at the end of the long waveguide wall shown in Fig. 1.

The center cell cavity shape parameters, which were used for optimization, are shown in Fig. 3. The optimization process resulted in a low $E_{p} / E_{\text {acc }}$ and $B_{p} / E_{\text {acc }}$, including the removal of the beam resonant frequency ( $2 N \times f_{\pi}, f_{\pi}$ is the fundamental mode frequency of the cavity) HOM. Other optimization parameters include beam pipe diameter and the slotted waveguide dimensions. By properly choosing the waveguide dimensions, the external quality factors $Q_{e}$ of the dipole and the quadrupole modes can be significantly reduced; and by optimizing the size of the beam pipe diameter, the monopole mode is damped to an acceptable value of $10^{5}$.

The center cell shape of the slotted cavities is based on the Argonne five-cell cavity. The center cell shape of the five-cell slotted cavity is exactly the same as the Argonne five-cell cavity. However, the center cell shape of the ninecell slotted cavity has been slightly modified to achieve low $E_{p} / E_{\text {acc }}$ and $B_{p} / E_{\text {acc }}$.

Parameters of the accelerating mode for the Argonne five-cell and the slotted cavities are shown in Table I. For completeness, the KEK-ERL model-2 nine-cell cavity parameters are also listed.

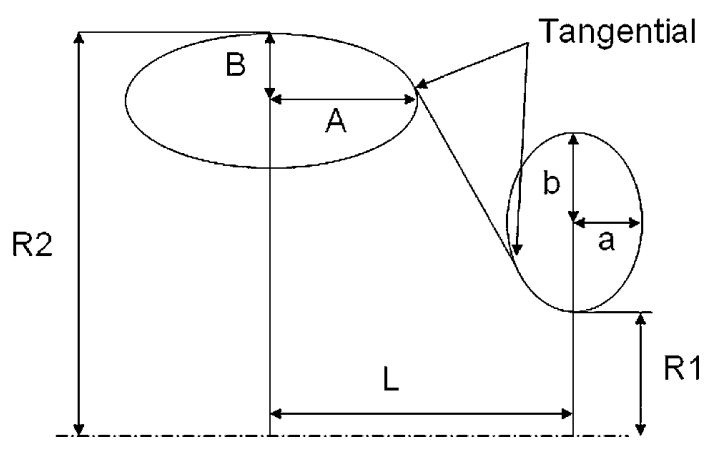

FIG. 3. Parameters for the center cell shape of the cavity. 
TABLE I. Parameters of the accelerating mode for the ANL and KEK-ERL cavities.

\begin{tabular}{lcccc}
\hline \hline Type & $\begin{array}{c}\text { Argonne } \\
\text { five-cell } \\
\text { cavity }\end{array}$ & $\begin{array}{c}\text { Five-cell } \\
\text { slotted } \\
\text { cavity } \\
\text { (unoptimized) }\end{array}$ & $\begin{array}{c}\text { Nine-cell } \\
\text { slotted } \\
\text { cavity } \\
\text { (optimized) }\end{array}$ & $\begin{array}{c}\text { KEK-ERL } \\
\text { model-2 } \\
\text { cavity }\end{array}$ \\
\hline Operating frequency $(\mathrm{MHz})$ & 1407.7 & 1406.1 & 1407.6 & 1300 \\
$Q_{0}$ & $9.3 \times 10^{9}$ & $9.3 \times 10^{9 \mathrm{a}}$ & $9.3 \times 10^{9}$ & $\ldots$ \\
Number of cell & 5 & 5 & 9 & 9 \\
$R / Q(\Omega)$ & 467 & 464 & 848.2 & 897 \\
$E_{p} / E_{\text {acc }}$ & 2.62 & 3.6 & 3 & 3.0 \\
$B_{p} / E_{\text {acc }}(\mathrm{mT} /(\mathrm{MV} / \mathrm{m}))$ & 4.19 & 4.7 & 4.7 & 4.25 \\
$Q_{0} \cdot R_{s}(\Omega)$ & 276 & $276^{\mathrm{a}}$ & 276 & 289 \\
Coupling factor $(\%)$ & 3.14 & 3.3 & 3.49 & 3.8 \\
\hline \hline
\end{tabular}

${ }^{\mathrm{a}}$ Using the numbers for the Argonne five-cell cavity.

\section{HOM IN THE CAVITY}

To achieve a high BBU threshold for multipass, multiturn ERLs utilizing superconducting cavities, it is essential to efficiently damp the dipole and the quadrupole modes without appreciably damping the accelerating mode $(\pi$ mode). The slotted cavity satisfies the HOM damping requirements of high-current SRF linac cavities. The dipole and the quadrupole modes of the slotted cavity are heavily damped without an appreciable damping of the cavity accelerating $\pi$ mode.

For the five-cell slotted cavity, we have chosen waveguide dimensions of $722.8 \mathrm{~mm} \times 10 \mathrm{~mm}$ to achieve low $E_{p} / E_{\text {acc }}$ and $B_{p} / E_{\text {acc }}$, large damping of the dipole and the quadrupole modes. The cutoff frequency for the waveguide mode $H_{10}$ (waveguide notation: the subscript 1 is in the long direction and 0 is in the short direction) is $207.5 \mathrm{MHz}$, which is much lower than the $\pi$ mode frequency of 1407.7 MHz. The cutoff frequency for $H_{01}$ is $15 \mathrm{GHz}$, which is very high compared to the major dipole modes of the cavity. Thus, we use the $H_{10}$ and its harmonics $\left(H_{n 0}\right.$, $n=1,2,3 \ldots)$ to propagate the HOM out of the cavity.
The mode, which has a strong transverse electric field close to the cavity wall, can easily excite $H_{n 0}(n=1,2,3 \ldots)$ in the waveguide. Therefore, such a mode can be propagated out from the waveguide. Fortunately, all the dipole and the quadrupole modes have strong transverse electric field close to the cavity wall with frequencies higher than the cutoff frequency. With the three waveguides around the center cavity, all the dipole and quadrupole modes of the slotted cavity can be propagated out through the waveguides. The monopole modes, however, cannot easily propagate through the waveguides. It is not easy to excite $H_{n 0}(n=1,2,3 \ldots)$ modes as it has no strong transverse electric field close to the wall. $H_{0 n}(n=1,2,3 \ldots)$ modes are easily excited by monopole modes; however, the cutoff frequency of the waveguides is $15 \mathrm{GHz}$, much higher than the main monopoles that are in the several-GHz range. Thus, we need to damp the monopole using absorbing materials inside of the end beam pipe instead. We have simulated and calculated the $Q_{e}$ of the HOM on the three waveguide ports. Figure 4 shows a high $R / Q$ of the dipole mode and weak electric fields on the end beam pipes. As can be seen, the fields in the waveguide are much stronger

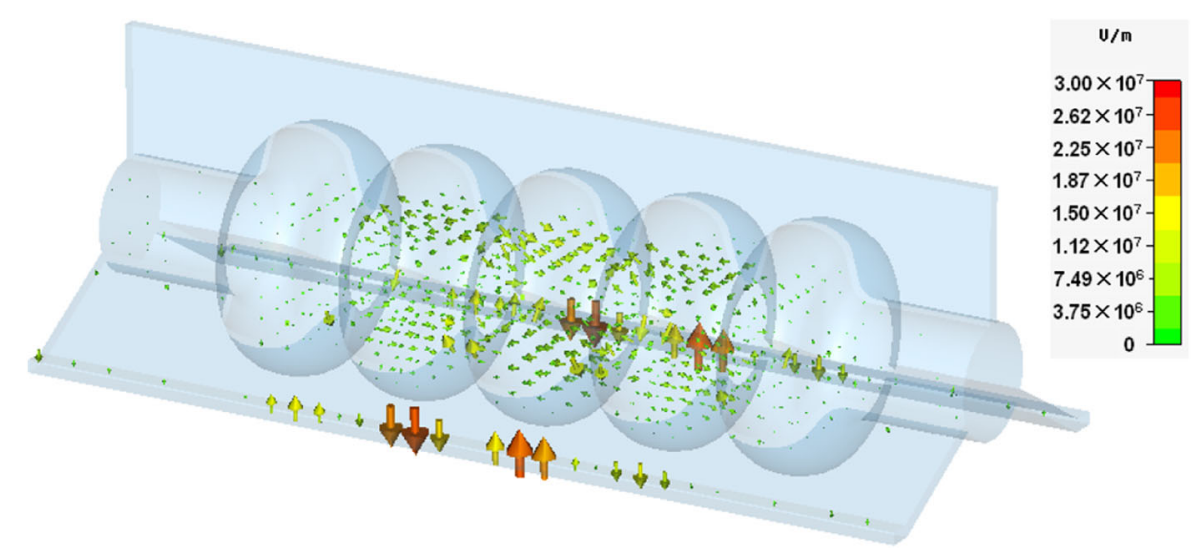

FIG. 4. (Color) $E$-field of a dipole mode in the slotted cavity. Frequency $=1.94799 \mathrm{GHz}, Q_{e}=137.8, R / Q=18.4 \Omega$. The electric field is derived by assuming $1 \mathrm{~J}$ stored energy of this dipole mode in the cavity. 
than the fields at the two ends, which will make it possible to be easily damped. Figure 5 shows a dipole that has the same strong field in each cell, which excites strong waves in the entire waveguide. Figure 6 shows a quadrupole mode in the cavity; this only excites strong waves in one waveguide due to the polarization angle. The presence of the waveguides located on the cavity, separated from each other by $120^{\circ}$, causes the quadrupole mode to propagate out by exciting a strong $H_{n 0}$ wave in the waveguide. Figure 7 shows the monopole $\pi$ mode in the slotted cavity. No strong field is excited in the waveguide, so the $Q_{e}$ of the accelerating $\pi$ mode at the waveguide ports is high. The $Q_{e}$ of the accelerating $\pi$ mode can be adjusted by moving the inner conductor of the coupler in or out. The designed

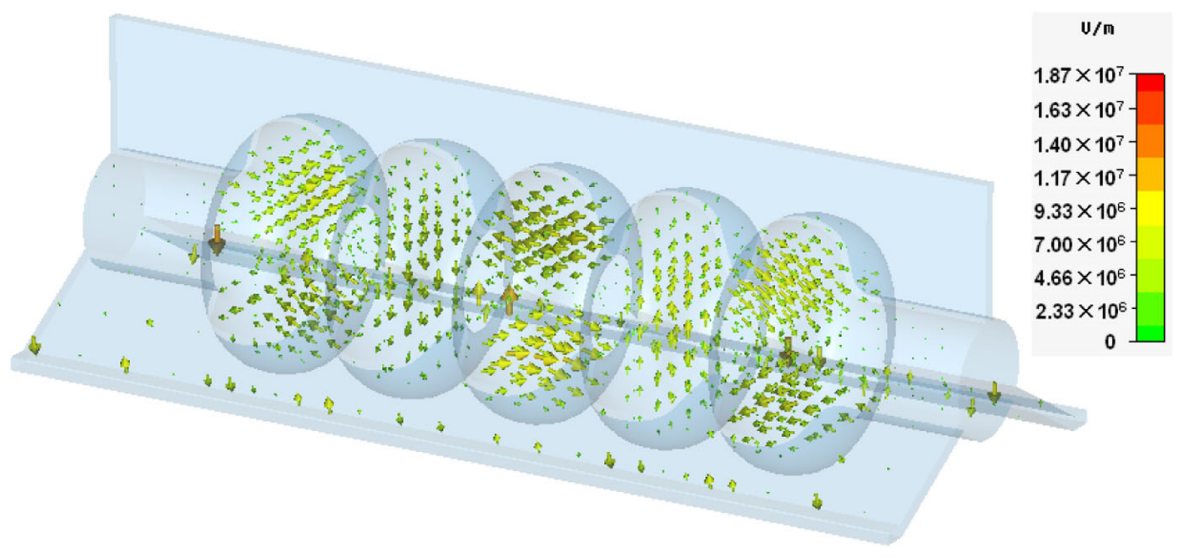

FIG. 5. (Color) $E$-field of a dipole mode in the slotted cavity. Frequency $=1.97299 \mathrm{GHz}, Q_{e}=1.02, R / Q=22.7 \Omega$. The electric field is derived by assuming $1 \mathrm{~J}$ stored energy of this dipole mode in the cavity.

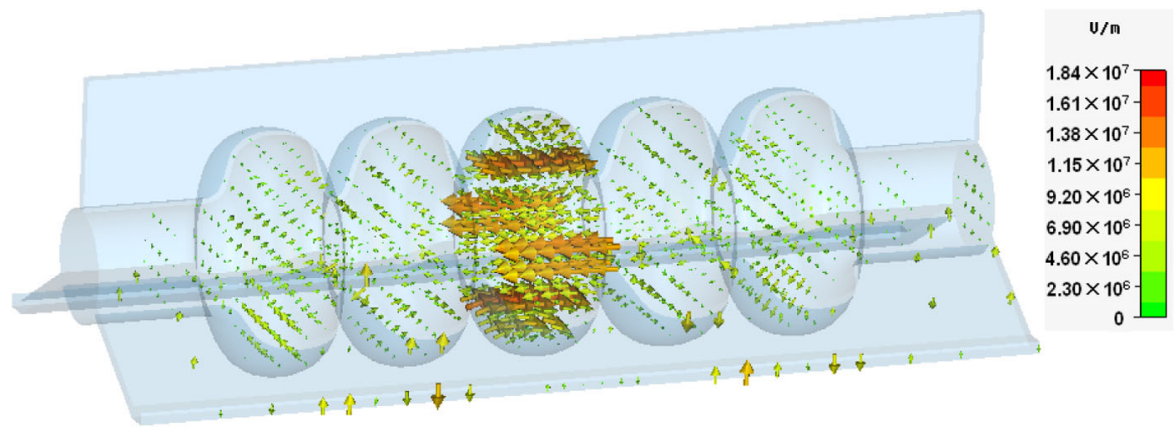

FIG. 6. (Color) $E$-field of a quadrupole mode with the highest $R / Q \cdot Q_{e} / f$ in the slotted cavity. Frequency $=2.63244 \mathrm{GHz}, Q_{e}=$ $379.2, R / Q=2.84 \times 10^{-2} \Omega / \mathrm{cm}^{4}$. The electric field is derived by assuming 1-J stored energy of this quadrupole mode in the cavity.

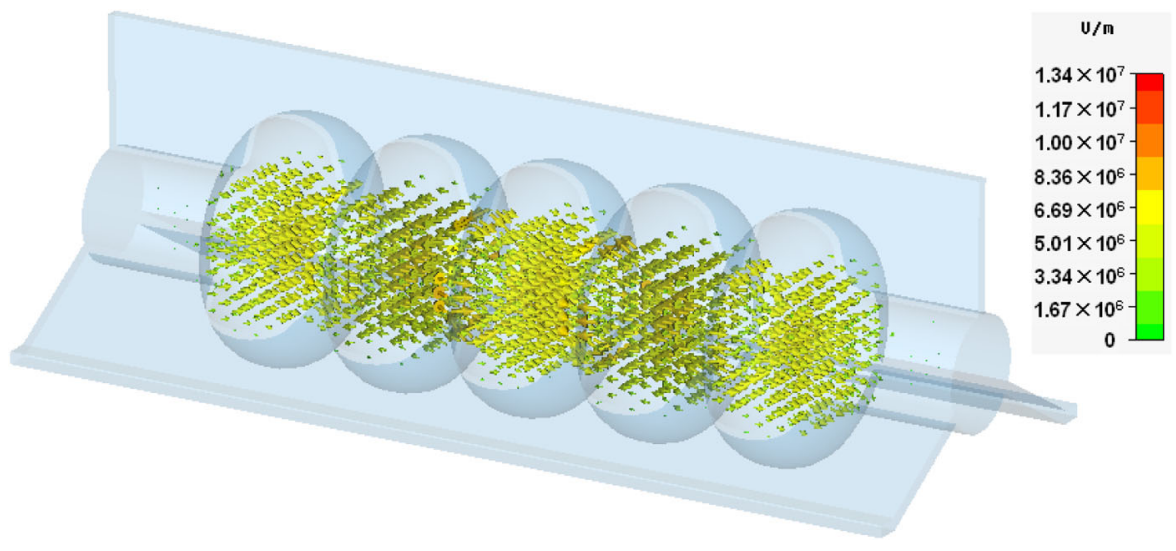

FIG. 7. (Color) $E$-field of the accelerating $\pi$ mode in the slotted cavity. Frequency $=1.4061 \mathrm{GHz}, Q_{e}=7.25 \times 10^{11}, R / Q=464 \Omega$. The electric field is derived by assuming $1 \mathrm{~J}$ stored energy of this $\pi$ mode in the cavity. 
TABLE II. $Q_{e}$ of the accelerating $\pi$ mode at the waveguide ports for different waveguide lengths.

\begin{tabular}{lc}
\hline \hline $\begin{array}{l}Q_{e} \text { of the accelerating } \\
\pi \text { mode at the waveguide ports }\end{array}$ & $\begin{array}{c}\text { Waveguide length } \\
\text { (mm, from equator) }\end{array}$ \\
\hline $3.79 \times 10^{6}$ & 14 \\
$6.58 \times 10^{7}$ & 19 \\
$7.91 \times 10^{8}$ & 24 \\
$1.12 \times 10^{11}$ & 29 \\
$1.31 \times 10^{11}$ & 34 \\
$7.25 \times 10^{11}$ & 54 \\
\hline \hline
\end{tabular}

tunable range of the $Q_{e}$ of the accelerating $\pi$ mode is from $10^{6}$ to $2 \times 10^{7}$. It can make the influence of microphonics controllable. The damping of $\pi$ modes at the waveguide ports of a five-cell slotted cavity was simulated with different waveguide lengths. Table II shows the $Q_{e}$ of the accelerating $\pi$ mode at the waveguide ports for different waveguide lengths. A prototype copper cavity will be fabricated to experimentally determine the optimum length of the extended waveguides, while keeping a high $Q_{e}$ of the accelerating mode. The experimental data will be compared to the simulation results.

We should point out that Chen [18] experimentally investigated a broadband damping of monopole, dipole, and quadrupole modes by implementing small longitudinal slots near the equatorial region of a single-cell copper cavity around 1990. The reported measurement results are consistent with our simulations and analysis.

Figure 8 shows the distribution of the cavity surface current for the accelerating $\pi$ mode, which is longitudi-

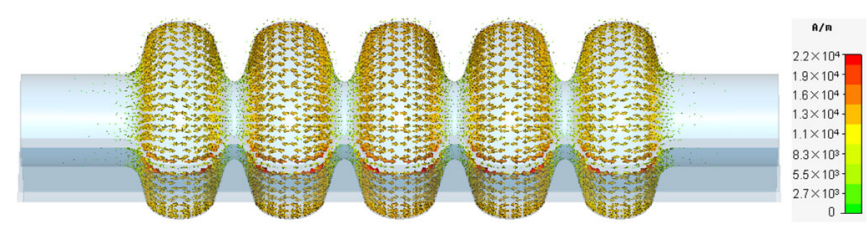

FIG. 8. (Color) Surface current profile of the accelerating $\pi$ mode of the slotted cavity.

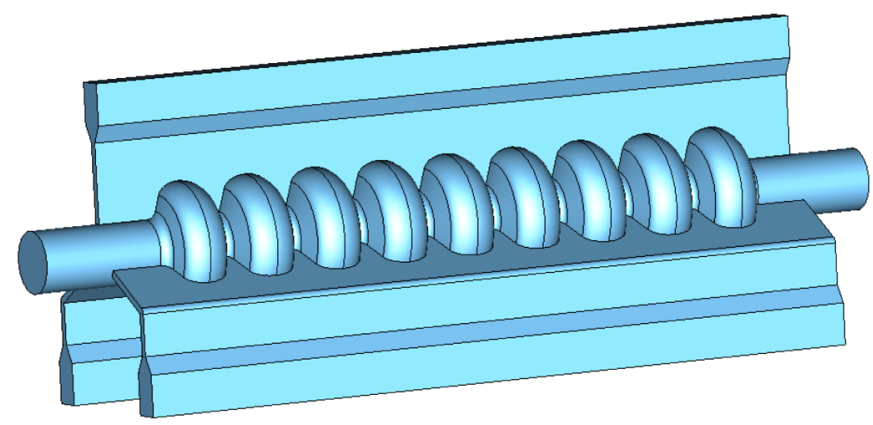

FIG. 9. (Color) Nine-cell slotted cavity model used in MWS simulation. The waveguide was bent parallel to the boundary for simulation. nally distributed; thus the presence of the slots, does not influence the accelerating $\pi$ mode. As can be seen from Fig. 8, the slots on the cavity make the electric field around the slot edges higher than at the other parts of the cavity surface. The five-cell slotted cavity has an $E_{p} / E_{\text {acc }}=3.6$, as compared to $E_{p} / E_{\text {acc }}=2.62$ of the Argonne five-cell cavity. It should be noted that the Argonne five-cell cavity was optimized to achieve a lower $E_{p} / E_{\text {acc }}$.

Since the waveguide slots extend from end to end, the trapped modes can be easily eliminated, which will allow us to incorporate a larger number of cells per structure to achieve a more efficient linac packing factor.

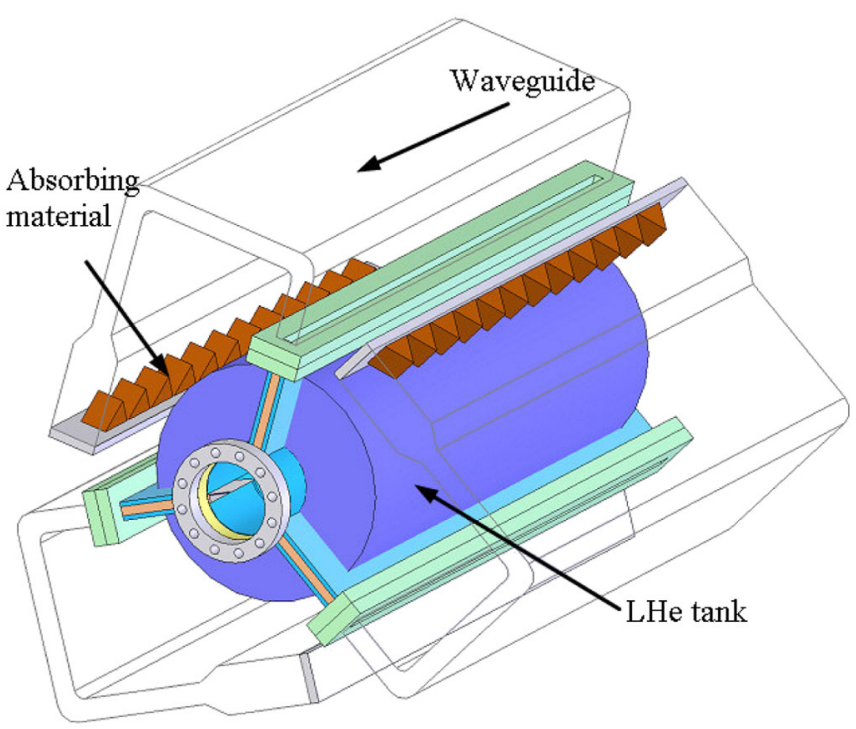

FIG. 10. (Color) Absorbing material (brown) position inside the waveguides.

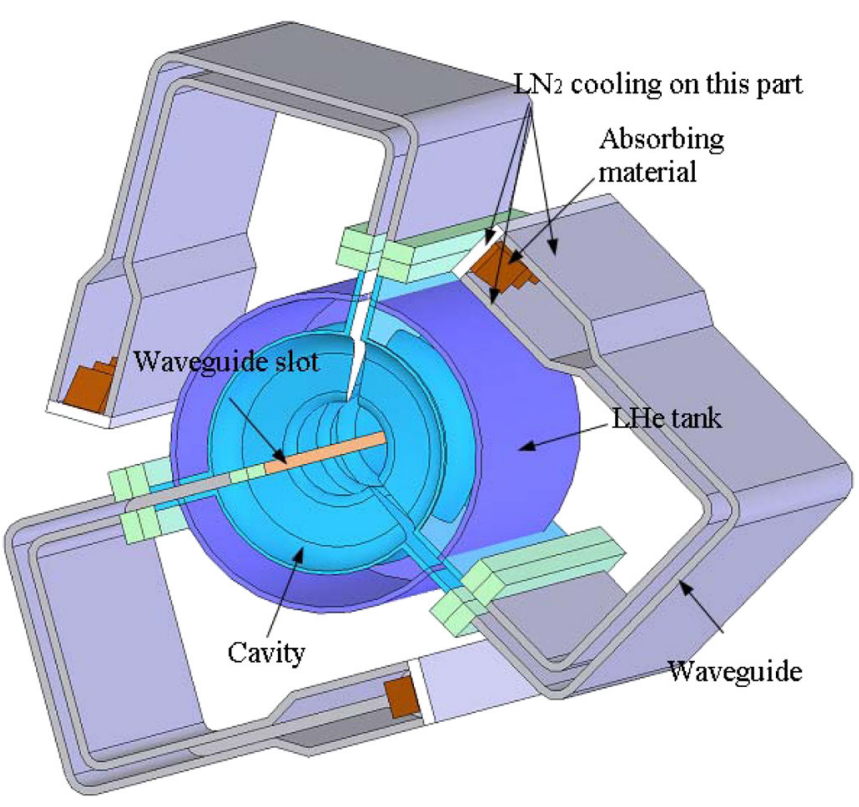

FIG. 11. (Color) Slotted cavity with waveguide attachments. 
As indicated above, for a fair comparison with other high-current cavities, we have designed a nine-cell slotted cavity. Optimization of the nine-cell slotted cavity has resulted in $E_{p} / E_{\text {acc }}=3.0$, which is acceptable for the accelerating field gradients in the range of 15 to $20 \mathrm{MV} / \mathrm{m}$. Figure 9 shows the nine-cell slotted cavity used for simulation. The ports for $Q_{e}$ calculation are at

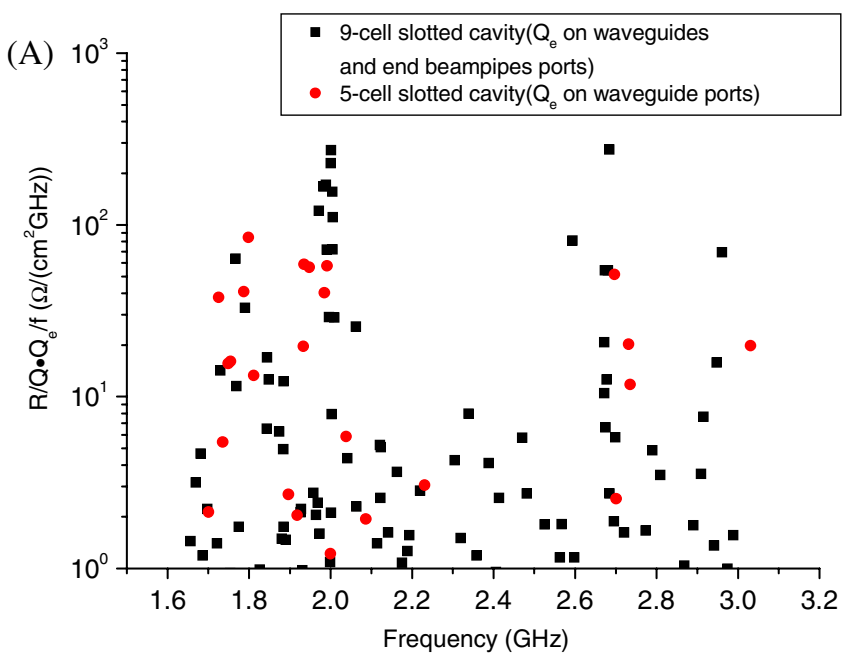

(B)
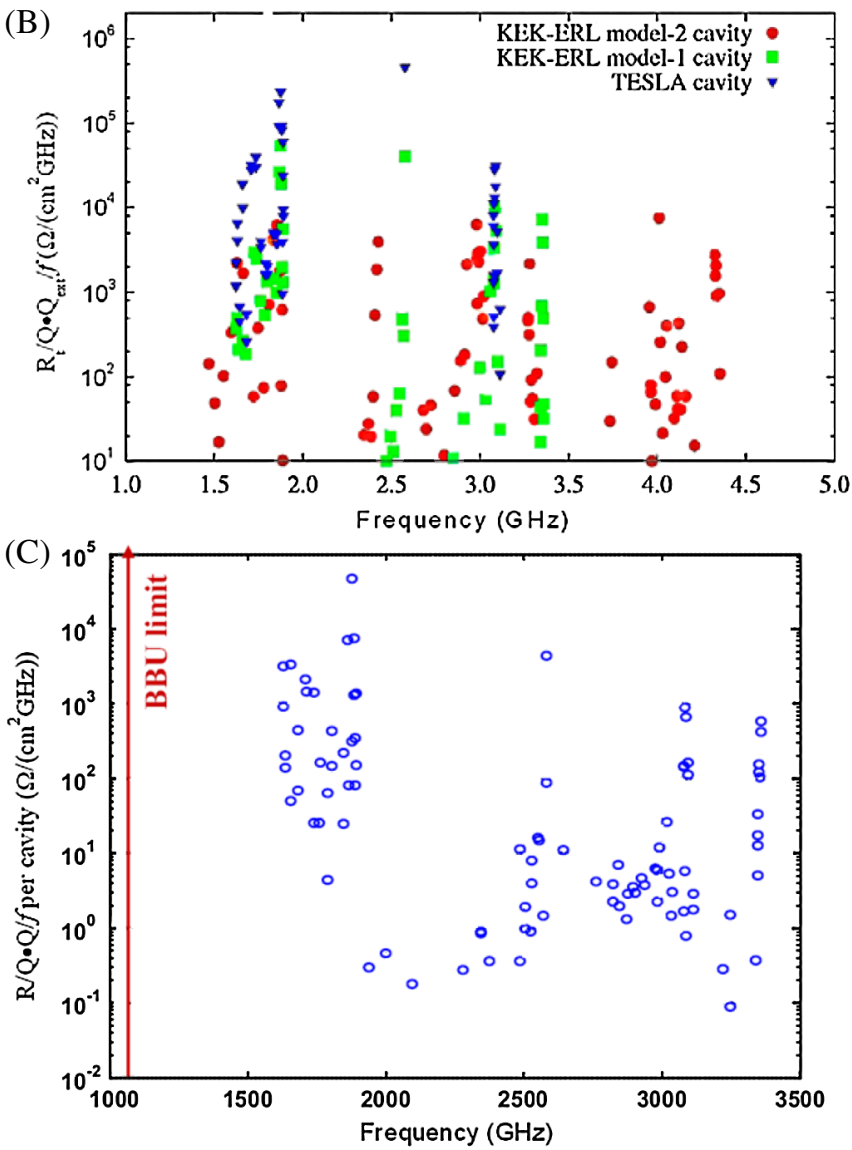

FIG. 12. (Color) A comparison of the dipole modes of three different cavities: (A) The slotted cavities; (B) KEK-ERL model-2 cavity [1]; (C) Cornell seven-cell cavity [4]. the ends of the waveguides and the beam pipes. Figure 10 shows the location and distribution of the absorbing material in the cavity. The HOM power can be absorbed in the cold region utilizing the absorbing material inside the waveguide. A schematic of the slotted structure with waveguides is shown in Fig. 11.

\section{HOM SIMULATION RESULTS}

The higher-order modes of the slotted cavities were simulated using MICROWAVE STUDIO (MWS). The rf performance of the waveguide-slotted cavities are compared to (1) the KEK-ERL model-2 cavity [1], and (2) the Cornell seven-cell cavity [4]. All of the above cavities are designed for high-beam-current (hundreds of milliamperes), multi-GeV ERLs. In comparison to the above cavities, the waveguide-slotted cavities show extremely good damping of the dipole and quadrupole modes. Figure 12 shows the distribution of the dipole modes of the cavities discussed above. For the nine-cell slotted cavity, the quan-
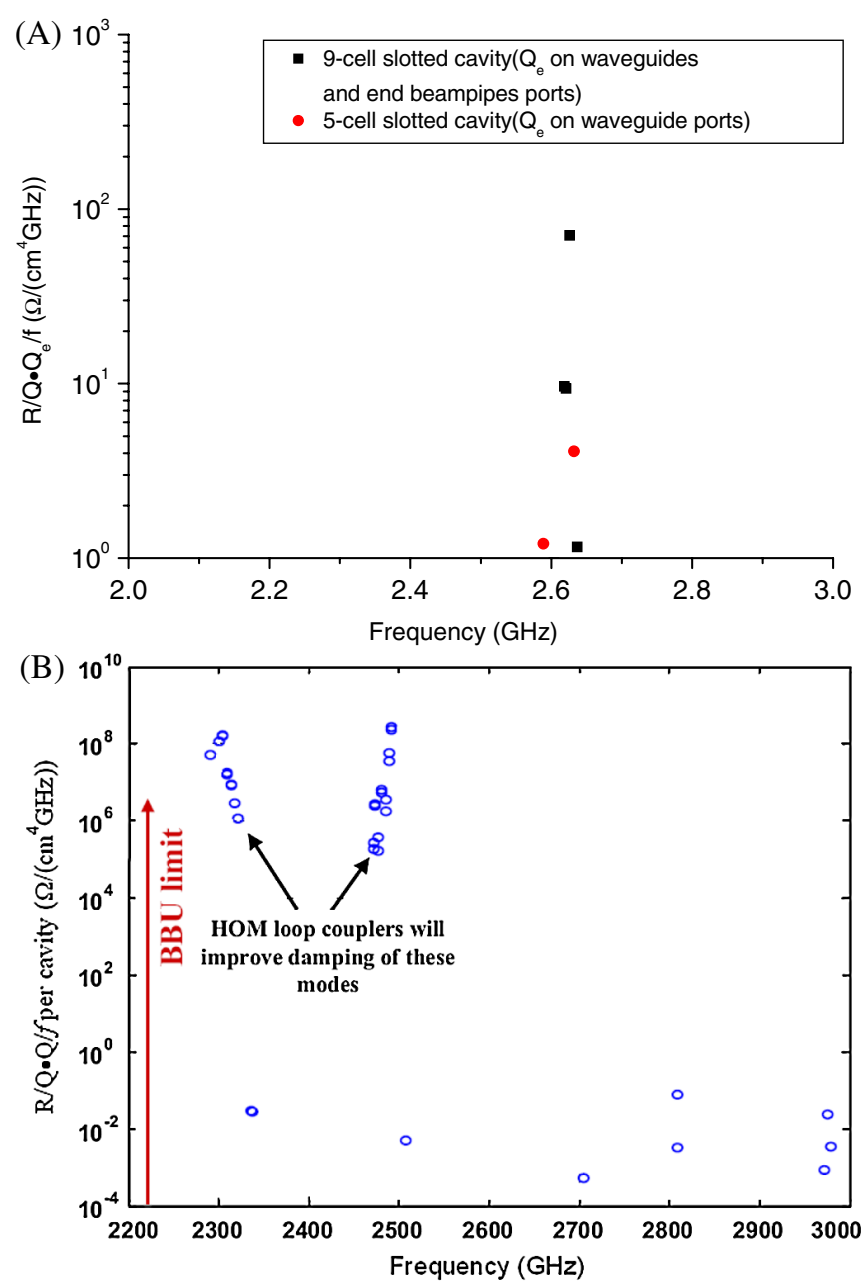

FIG. 13. (Color) Comparison of the quadrupole modes for two different cavities: (A) Slotted cavity; (B) Cornell seven-cell cavity [4]. 


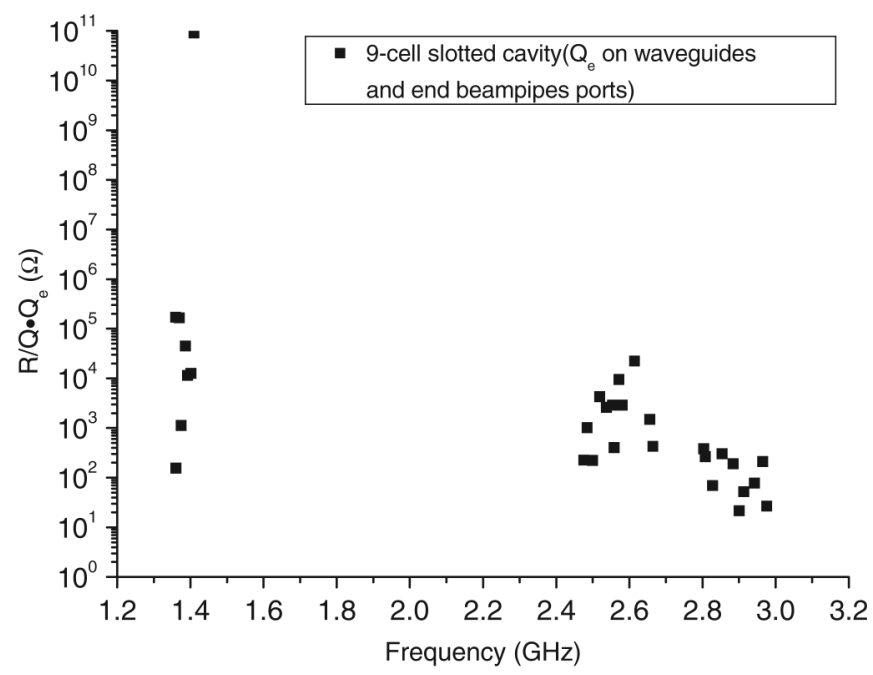

FIG. 14. Monopole modes of the nine-cell slotted cavity.

tity $R / Q \cdot Q_{e} / f$ of the dipole modes is under $300 \Omega /\left(\mathrm{cm}^{2} \mathrm{GHz}\right)$. The dipole mode damping of the nine-cell slotted cavity is an order of magnitude lower than the KEK-ERL model- 2 cavity and 2 orders of magnitude lower than the Cornell seven-cell cavity. The quadrupole modes distribution of the slotted cavities is shown in Fig. 13. The $R / Q \cdot Q_{e} / f$ of all quadrupole modes is under $10^{2} \Omega /\left(\mathrm{cm}^{4} \mathrm{GHz}\right)$ for the nine-cell slotted cavity, which is roughly 6 orders of magnitude better than the Cornell seven-cell cavity.

Figure 14 shows the monopole damping of the nine-cell slotted cavity. The impedance, $R / Q \cdot Q_{e}$ of the accelerating mode, is about $10^{11} \Omega$, while the impedance of other monopole modes is below $3 \times 10^{5} \Omega$.

\section{BBU THRESHOLD}

The BBU threshold of the cavity is predicted by the computer program TDBBU using the major dipole modes of the cavity. The simulation is performed for the conceptual APS single-pass 7-GeV ERL with the final decelerated beam energy of $10 \mathrm{MeV}$ and a nominal $20 \mathrm{MV} / \mathrm{m}$ accelerating gradient. Figure 15 shows the simulated results of a 1.5-A accelerating beam using nine-cell slotted cavities. The vertical axis of each figure is the displacement of the bunch leaving the accelerator as a function of the bunch number. As can be seen in Fig. 15, the displacements of the bunch in the $x$ and $y$ directions of the cavity converge separately. Thus, a BBU threshold of $1.5 \mathrm{~A}$ for the APS 7-GeV ERL can be achieved using nine-cell slotted cavities.

\section{CAVITY FABRICATION AND CLEANING PROCESS}

Fabrication processes determine the cost and performance of a superconducting cavity. In comparison with the traditional design and fabrication of the superconducting
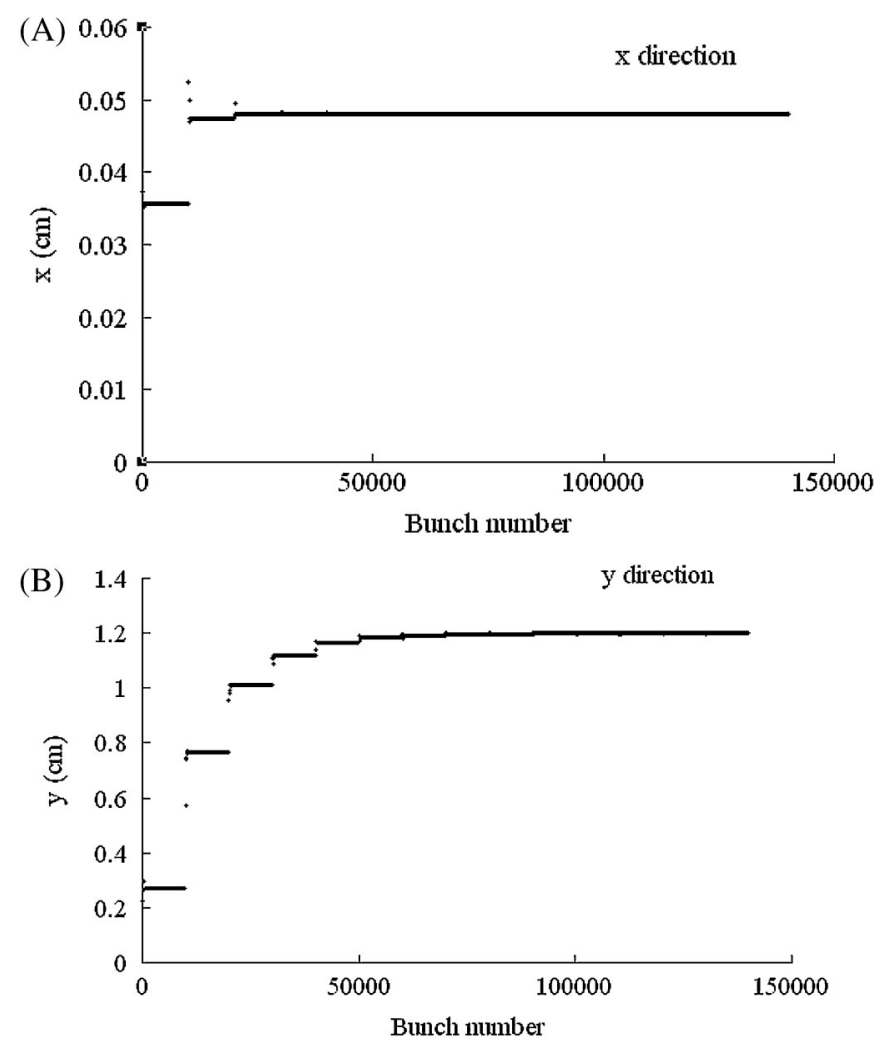

FIG. 15. BBU threshold simulation of slotted cavity. Beam current is $1.5 \mathrm{~A}$ (accelerating beam). Injected energy $=$ $10 \mathrm{MeV}, \quad E_{\mathrm{acc}}=20 \mathrm{MV} / \mathrm{m}$, final electron-beam energy = $7 \mathrm{GeV}$. The vertical axes of the two plots are the displacement of the bunch leaving the accelerator as a function of bunch number: (A) $x$ direction of the cavity (horizontal); (B) $y$ direction of the slotted cavity (vertical).

cavities, the slotted cavity does not require welding at the high-field regions. The cavity body with two waveguide walls will be deep drawn to form a one-third portion of the cavity. The three equal sections will then be welded together as shown in Fig. 1. Simulations show that a smaller waveguide edge radius produces a much smaller perturbation on the fundamental accelerating mode field pattern. Therefore, for the copper prototype cavity, we will choose a smaller radius of roughly $0.5 \mathrm{~mm}$. Eliminating these welds will not only result in a streamlined welding process and reduction in fabrication time, it will also provide an appreciable reduction in fabrication cost. In addition, this design will eliminate field emission and quench caused by the welds on the high-field regions, which is problematic in conventional fabrication processes. Another key advantage of the slotted cavity is its simple fabrication process, which will only require deep drawing of a single niobium sheet to form a $1 / 3$ of the cavity shape.

We believe that a high-pressure ( $\sim 80-100$ bar) rinse with ultrapure water can be effectively adapted for the slotted cavities with waveguide extensions. An on-axis rinsing tube can be used to clean contaminants from the surface of the cavities, while an appropriately devised 
manual cleaning method can be employed to clean the inner surface of the 1 -cm-wide, 5-cm-long waveguide extensions from the outside open waveguide ports. We plan to further explore current SRF-cavity surface cleaning processes as applied to the slotted waveguide structures proposed here.

To address the multipacting issues inside the slotted waveguide extensions, we plan to perform multipacting simulation and analysis using TRACK3P [19] and fabricate a niobium cavity to perform cold tests at the temperature of $2 \mathrm{~K}$.

\section{CONCLUSIONS}

The slotted cavity design presented here is based on a previous Argonne five-cell superconducting cavity design, which was optimized for the proposed APS 7-GeV ERL. The new slotted cavity provides the necessary damping of the dipole and quadrupole modes without adversely damping the accelerating $\pi$ mode. This design has significant advantages over the traditional elliptical multicell cavities, as it makes damping of the dipole and quadrupole modes easier and more effective. We have demonstrated that the trapped modes inside the cavity are eliminated, thus allowing a larger number of cells per structure for a better accelerator packing factor. The fabrication steps and processes are significantly simplified, resulting in a reduction of the fabrication time and cost. We have shown that the slotted cavity will fulfill the operational requirements of the ampere-class multi-GeV ERLs. More studies and design optimizations are underway.

\section{ACKNOWLEDGMENTS}

This work was supported by the U.S. Department of Energy, Office of Science, Office of Basic Energy Sciences, under Contract No. DE-AC02-06CH11357. Z. Liu was sponsored by the China Scholarship Council.

[1] T. Furuya et al., in Proceedings of the Energy Recovery Linac Workshop (ERL07) (Daresbury Laboratory, Daresbury, 2007), p. 56, paper 34; http://www.JACoW.org.

[2] Z. Liu et al., in Proceedings of the Energy Recovery Linac Workshop (ERL09) (Cornell University, Ithaca, NY, 2009).
[3] I. Ben-Zvi, Physica C (Amsterdam) 441, 21 (2006).

[4] M. Liepe, in Proceedings of the Eleventh Workshop on RF Superconductivity (SRF03) (Travemunde/Lubeck, Germany, 2003), paper MoP33.

[5] H. Wang, R. A. Rimmer, and F. Marhauser, in Proceedings of the 2007 Particle Accelerator Conference, Albuquerque, New Mexico, 2007 (IEEE, Albuquerque, New Mexico, 2007), p. 2496, paper WEPMS070; http:// www.JACoW.org.

[6] F. Marhauser et al., in Proceedings of the 11th European Particle Accelerator Conference, Genoa, 2008 (EPS-AG, Genoa, Italy, 2008), p. 886, paper MOPP140; http:// www.JACoW.org.

[7] R. Calaga et al., in Proceedings of the 9th European Particle Accelerator Conference, Lucerne, 2004 (EPSAG, Lucerne, 2004), p. 1227, paper TUPKF078; http:// www.JACoW.org.

[8] V. N. Litvinenko et al., in Proceedings of the 21st Particle Accelerator Conference, Knoxville, 2005 (IEEE, Piscataway, NJ, 2005), p. 2242, paper RPPT032; http:// www.JACoW.org.

[9] B. Aune et al., Phys. Rev. ST Accel. Beams 3, 092001 (2000).

[10] K. Beard, L. Merminga, and B. Yunn, in Proceedings of the 2007 Particle Accelerator Conference, Albuquerque, New Mexico, 2007 (Ref. [5]), p. 332, paper TOPD006; http://www.JACoW.org.

[11] J. Knobloch, IEEE Trans. Appl. Supercond. 9, 1016 (1999).

[12] G. Hoffstaetter, I. Bazarov, and C. Song, Phys. Rev. ST Accel. Beams 10, 044401 (2007).

[13] E. Pozdeyev, Phys. Rev. ST Accel. Beams 8, 054401 (2005).

[14] G. Hoffstaetter and I. Bazarov, Phys. Rev. ST Accel. Beams 7, 054401 (2004).

[15] C. Song and G. Hoffstaetter, in Proceedings of the 2007 Particle Accelerator Conference, Albuquerque, New Mexico, 2007 (Ref. [5]), p. 1227, paper TUPMS022; http://www.JACoW.org.

[16] M. Borland et al., Ref. [2].

[17] M. Borland et al., Ref. [2].

[18] Y. Chen, D. Proch, and J. Sekutowicz, in Proceedings of the 14th International Conference on High Energy Accelerators, Tsukuba, Japan (Gordon and Breach, New York, 1989); Part. Accel. 29, 741 (1990).

[19] Z. Li et al., in Proceedings of the 2007 Particle Accelerator Conference, Albuquerque, New Mexico, 2007 (Ref. [5]), p. 889, paper TUZBC01. 